\title{
Colonisation resistance in the sand fly gut: Leishmania protects Lutzomyia longipalpis from bacterial infection
}

Mauricio RV Sant'Anna ${ }^{1,2}$, Hector Diaz-Albiter ${ }^{3}$, Kelsilândia Aguiar-Martins ${ }^{4}$, Waleed S Al Salem ${ }^{5}$, Reginaldo R Cavalcante ${ }^{6}$, Viv M Dillon? ${ }^{7}$ Paul A Bates ${ }^{1}$, Fernando A Genta ${ }^{3}$ and Rod J Dillon ${ }^{1^{*}}$

\begin{abstract}
Background: Phlebotomine sand flies transmit the haemoflagellate Leishmania, the causative agent of human leishmaniasis. The Leishmania promastigotes are confined to the gut lumen and are exposed to the gut microbiota within female sand flies. Here we study the colonisation resistance of yeast and bacteria in preventing the establishment of a Leishmania population in sand flies and the ability of Leishmania to provide colonisation resistance towards the insect bacterial pathogen Serratia marcescens that is also pathogenic towards Leishmania.

Methods: We isolated microorganisms from wild-caught and laboratory-reared female Lutzomyia longipalpis, identified as Pseudozyma sp. Asaia sp. and Ochrobactrum intermedium. We fed the females with a sugar meal containing the microorganisms and then subsequently fed them with a bloodmeal containing Leishmania mexicana and recorded the development of the Leishmania population. Further experiments examined the effect of first colonising the sand fly gut with L. mexicana followed by feeding with, Serratia marcescens, an insect bacterial pathogen. The mortality of the flies due to $S$. marcescens was recorded in the presence and absence of Leishmania.

Results: There was a reduction in the number of flies harbouring a Leishmania population that had been pre-fed with Pseudozyma sp. and Asaia sp. or O. intermedium. Experiments in which L. mexicana colonised the sand fly gut prior to being fed an insect bacterial pathogen, Serratia marcescens, showed that the survival of flies with a Leishmania infection was significantly higher compared to flies without Leishmania infection.

Conclusions: The yeast and bacterial colonisation experiments show that the presence of sand fly gut microorganisms reduce the potential for Leishmania to establish within the sand fly vector. Sand flies infected with Leishmania were able to survive an attack by the bacterial pathogen that would have killed the insect and we concluded that Leishmania may benefit its insect host whilst increasing the potential to establish itself in the sand fly vector. We suggest that the increased ability of the sand fly to withstand a bacterial entomopathogen, due to the presence of the Leishmania, may provide an evolutionary pressure for the maintenance of the Leishmania-vector association.
\end{abstract}

Keywords: Leishmania, Lutzomyia, Asaia, Pseudozyma, Serratia, Sand fly, Microbiota

\footnotetext{
* Correspondence: R.dillon@lancaster.ac.uk

${ }^{1}$ Faculty of Health and Medicine, Division of Biomedical and Life Sciences,

Lancaster University, Lancaster, UK

Full list of author information is available at the end of the article
} 


\section{Background}

Female phlebotomine sand flies are the primary vectors for transmission of the medically important haemoflagellate Leishmania between their mammalian hosts $[1,2]$. Leishmania develop exclusively within the gut of the female insect [3] and this means that Leishmania will be exposed to other microorganisms that are either resident or passing through the gut. Adult sand flies acquire their microbiota from several sources: during blood feeding on their animal hosts; from plants on which the adults feed and via the pupal stage from exposure of larval stages to their terrestrial dwelling sites [4-8].

The concept of colonisation resistance is well established in vertebrate gut systems [9]. This is the ability of the gut microbiota to prevent the development of invasive microorganisms, commonly potential pathogens, by a combination of direct microbial interaction and indirect actions of the mucosal immune system. A similar concept has been presented for insects [10]. A role for insect gut bacteria in colonisation resistance towards medically important parasites has been established in other insects [10-14]. In these studies, the gut microbiota may reduce transmission of the medically important parasite rather than prevent development of an insect pathogen. Nearly 30 years ago, Schlein et al. discussed the possibility of gut microorganisms in Phlebotomine sand flies preventing Leishmania development [15]. In the first part of our study, we explored the effects of yeast and bacterial colonisation of the female sand fly gut on the subsequent establishment of Leishmania in the gut of the vector. The microorganisms used for the investigation were the bacteria Asaia sp., Ochrobactrum intermedium and a yeastlike fungus Pseudozyma sp. from the gut of field-caught or lab-reared Lu. longipalpis.

In the second part of our study, we addressed the idea that the Leishmania might promote colonisation resistance for the sand fly host. Adult sand flies are short lived, averaging perhaps 10 days in the wild [16]. For successful transmission during that time the sand fly must first feed on a Leishmania-infected mammal to acquire the parasites, these must then colonise the gut, and the insect must remain healthy for long enough to transfer Leishmania to another mammal when the Leishmania is regurgitated during the next blood feed [17]. The benefit for Leishmania in its relationship with the insect is obvious, but there are fitness costs for the sand fly: Leishmania populations in the gut can lead to a reduction in Lu. longipalpis longevity and this is effect is enhanced if infected flies are stressed [3,18]. It is known that Leishmania can modulate the digestive enzymes of the sand fly host [19-21] and may cause damage to the stomodeal valve in the gut [22]. There is also evidence for an insect immune response towards Leishmania and if the sand fly immune system is appropriately stimulated this can lead to a loss of Leishmania infection [23]. However, the idea that a Leishmania infection may be of advantage to the sand fly has never been explored; even intermittent benefits of Leishmania towards its vector will have a profound influence on the relationship. In the second part of the study we designed experiments to explore the potential benefits to the sand fly host in harbouring Leishmania in the gut. We tested the hypothesis to see if the presence of Leishmania could provide colonisation resistance towards entomopathogenic bacteria. These experiments were performed using the sand fly Lu. longipalpis [24] together with Leishmania mexicana, an excellent model to study Leishmania-sand fly interactions [17], and Serratia marcescens an insect bacterial pathogen. This bacterium was previously found associated with wild $L u$. longipalpis [7] and is also lethal to Leishmania in vitro [25].

\section{Methods}

\section{Ethics statement}

All procedures involving animals were approved by the ethical review committee of the University of Liverpool and performed in accordance with United Kingdom Government (Home Office approved project licence PPL40/ 2958) and EC regulations.

\section{Sand fly rearing}

All experiments were performed using $\mathrm{Lu}$. longipalpis obtained originally from Jacobina (Bahia-Brazil) and reared under standardised laboratory conditions [26]. Adult insects were kept under controlled temperature $\left(27 \pm 2^{\circ} \mathrm{C}\right)$, humidity (> 80\%) and photoperiod ( 8 hours light/16 hours darkness) and were fed on a diet consisting of autoclaved $70 \%$ w/v sucrose solution on cotton wool ad libitum. Female sand flies were blood fed once a week with EDTAtreated rabbit blood via a Hemotek membrane feeder (Discovery Workshops-UK) at $37^{\circ} \mathrm{C}$.

\section{Isolation of microorganisms from sand flies}

Wild-caught $L u$. longipalpis were captured in Teresina, an endemic area under active urban transmission of visceral leishmaniasis in Northeast Brazil. The midgut from each single surface sterilised fly $(70 \% \mathrm{v} / \mathrm{v}$ ethanol for 3 mins) was homogenised in $50 \mu \mathrm{l}$ of PBS and serial dilutions were inoculated onto LB/agar plates and incubated at $26^{\circ} \mathrm{C}$ for $24-72 \mathrm{~h}$. Selected colonies of field-isolated bacteria and yeast-like fungi were identified by PCR amplification using primers for the 16 or 26S rRNA gene. Full-length sequences of the 16S rRNA gene were obtained for field-isolated bacteria Asaia sp. (HE995765) and Ochrobactrum intermedium (HE995764) isolated from our laboratory colony $\mathrm{Lu}$. longipalpis using previously described primers [27]. A yeast-like fungus Pseudozyma sp (KJ493325) was identified from a wild caught sand fly using primers NL-1 and NL-4, designed for yeasts, for the 
variable D1/D2 domain of the large subunit (26S) ribosomal DNA sequence [28].

\section{Sand fly infections}

Sand fly infections with promastigotes of $L$. mexicana (strain MNYC/BZ/62/M379) were performed as described previously [29]. Heat-inactivated $\left(56^{\circ} \mathrm{C}\right.$ for 1 hour) rabbit blood was used to re-suspend cultured promastigotes to a final concentration of $1 \times 10^{6}$ parasites $\mathrm{mL}^{-1}$ in comparison to a control without heat inactivation. Rabbit blood seeded with parasites was offered to Lu. longipalpis 96 hours after bacterial or yeast feed. Infected sand flies were then transferred to new cages, dissected 72 hours after infection and the number of Leishmania promastigotes inside their midguts was recorded using a haemocytometer.

\section{Colonisation resistance experiments}

Pseudozyma (strain Pa1), Asaia sp. (A1) and Ochrobactrum intermedium (Om17) were inoculated on GLY/agar plates (contains nutrients $2.5 \% \mathrm{w} / \mathrm{v}$ glycerol and $1 \% \mathrm{w} / \mathrm{v}$ yeast extract) or Luria-Bertani agar (O. intermedium) and incubated overnight for 24 hours at $26^{\circ} \mathrm{C}$. Single colonies were transferred to media in $50 \mathrm{~mL}$ polypropylene tubes and grown in a shaking incubator (200 rpm). Growth conditions were; $18 \mathrm{~h}$ in GLY media $(\mathrm{pH}=5.5)$ at $30^{\circ} \mathrm{C}$ (Pseudozyma sp.) and $72 \mathrm{~h}$ (Asaia sp.); $18 \mathrm{~h}$ in $40 \mathrm{~mL}$ of LB media at $37^{\circ} \mathrm{C}$ (O. intermedium). The microbial suspensions were centrifuged at $6,500 \mathrm{~g}$ for 5 minutes and re-suspended in $0.22 \mu \mathrm{m}$-filtered $7 \%$ sucrose solution to a concentration between 2- $4 \times 10^{7} \mathrm{CFU} \mathrm{mL}^{-1}$. When Pseudozyma and Asaia were fed in combination the total concentration was also adjusted to $2-4 \times 10^{7} \mathrm{CFU} \mathrm{mL} \mathrm{m}^{-1}$. The microbial suspensions were fed to 3 day old sand flies via a Hemotek membrane through chick skin feeders. Control flies were fed on sterile 7\% sucrose solution. Membrane feeding of bacteria and selection of only fully engorged insects was done to standardise the amount of bacteria ingested. Fully engorged female sand flies were allowed to rest for $96 \mathrm{~h}$, prior to Leishmania infection using $10^{6}$ promastigotes per $\mathrm{mL}$ in heat-inactivated rabbit blood. Sand flies were dissected at $72 \mathrm{~h}$ after Leishmania infection and the numbers of promastigotes were counted using a haemocytometer.

To investigate the effect of dose on colonisation resistance towards Leishmania an O. intermedium culture was grown overnight in Luria-Bertani medium and diluted to $1 \times 10^{6}$ and $1 \times 10^{7} \mathrm{CFU} \mathrm{mL} \mathrm{m}^{-1}$. The bacterial suspensions were prepared and fed to flies as described above. The effect of live bacteria on colonisation resistance was assessed by pre-feeding heat inactivated O. intermedium $\left(80^{\circ} \mathrm{C}\right.$ for 15 minutes with a $10^{7} \mathrm{CFU} \mathrm{mL}{ }^{-1}$ culture) and monitoring Leishmania development within the sand fly gut in comparison to a live O. intermedium culture at the same concentration.
Incubation of $L$. mexicana with yeast and bacteria in vitro Pseudozyma sp, Ochrobactrum intermedium and Asaia sp. were inoculated in liquid LB media and incubated overnight. Cells without media were obtained by centrifuging the cultures at $6,500 \mathrm{~g}$ for 5 minutes and washing cells twice with sterile PBS. Culture media free of cells was obtained by filtering cultures with $0.22 \mu \mathrm{M}$ filters prior to incubation with Leishmania. L. mexicana promastigotes were centrifuged at 2,000 g for 5 minutes, washed twice with sterile $1 \times \mathrm{PBS}$ and re-suspended to $6 \times 10^{6}$ promastigotes $\mathrm{mL}^{-1}$. A 1:1 mixture of L. mexicana promastigotes $\left(3 \times 10^{6}\right.$ final concentration) were incubated either with cells $\left(10^{7} \mathrm{CFU} \mathrm{mL}{ }^{-1}\right.$ final concentration) or previously used culture media at $26^{\circ} \mathrm{C}$ for $24 \mathrm{~h}$. The number of surviving promastigotes was recorded using a haemocytometer.

\section{L. mexicana incubation with S. marcescens in vitro}

The $L$. mexicana promastigotes were centrifuged at $1,500 \mathrm{~g}$ for 10 minutes, washed twice with sterile PBS and resuspended in PBS. Serratia marcescens was inoculated in liquid Luria-Bertani media and grown overnight as described above. After a centrifugation at $6,500 \mathrm{~g}$ for $5 \mathrm{mi}-$ nutes, culture media was discarded; bacterial cells were washed twice in PBS. $1 \mathrm{~mL}$ of re-suspended bacterial cells (final concentration of $10^{7} \mathrm{CFUmL}^{-1}$ ) was incubated overnight with $1 \mathrm{~mL}$ of Leishmania promastigotes (final concentration $3 \times 10^{6}$ parasites $\mathrm{mL}^{-1}$ ) at $26^{\circ} \mathrm{C}$. Incubations were repeated three times in triplicate and the number of Leishmania promastigotes was recorded using a haemocytometer at 24 hours after incubation. For incubations with culture supernatant the $S$. marcescens was inoculated in liquid Luria-Bertani media and grown overnight at $37^{\circ} \mathrm{C}$ (200 rpm). Spent medium from Serratia culture was produced by filtering $(0.22 \mu \mathrm{m}$ filter $)$ a bacterial culture $\left(5.7 \times 10^{7} \mathrm{CFU} \mathrm{mL}{ }^{-1}\right)$. The spent media and parasites were incubated together and assessed as described above.

\section{Leishmania DNA quantification by $\mathrm{qPCR}$}

Leishmania DNA quantification in adult females of $L u$. longipalpis was performed as previously described [30]. Briefly, $50 \mathrm{ng}$ of sand fly DNA from 12 pools of 5 insects was used in a real time PCR reaction using primers that amplify a 120-bp fragment of the Leishmania minicircle kDNA. A standard curve was constructed using DNA from L. mexicana (strain MNYC/BZ/62/M379) obtained from promastigote cultures $\left(3 \times 10^{7} \mathrm{~mL}^{-1}\right)$ in a 10 fold dilution series ranging from $10 \mathrm{ng}$ to $100 \mathrm{fg}$.

\section{Bacterial quantification in laboratory reared sand flies}

Detection of bacterial DNA by quantitative PCR was performed as previously described [23]. Briefly, 6 pools of 5 newly emerged female $\mathrm{Lu}$. longipalpis (approximately $1 \mathrm{~h}$ after emergence), 3 day old flies sugar fed with sterile $70 \%$ 
sucrose solution changed daily and blood fed flies at 1, 24 and $72 \mathrm{~h}$ after blood feed were surface-sterilised in $70 \%$ ethanol for $1 \mathrm{~min}$ and rinsed in $1 \times$ PBS for $1 \mathrm{~min}$. Total DNA was extracted, diluted to a final concentration of $50 \mathrm{ng}$ of DNA $\mathrm{Il}^{-1}$ and added to the quantitative PCR mix. Bacterial copy numbers were calculated using Asaia DNA as standard and universal bacterial primers [31].

\section{Leishmania and Serratia co-infection of sand flies}

Female sand flies were infected with L. mexicana 3 days after emergence as described above. Infected sand flies were transferred to a new cage and maintained with autoclaved $70 \% \mathrm{w} / \mathrm{v}$ sucrose solution on cotton wool for 4 days. Leishmania-infected sand flies were then orally challenged with Serratia, re-suspended to give a concentration of $5.7 \times 10^{7} \mathrm{cfu} \mathrm{mL}^{-1}$ in autoclaved $20 \% \mathrm{w} / \mathrm{v}$ sucrose solution and fed to Leishmania-infected sand flies daily via cotton wool. Cotton wool moistened with Serratia solution was changed daily and sand fly survival was monitored for 6 days after bacterial challenge and compared with uninfected bloodfed sand flies that were also challenged with Serratia. Additional control groups consisted of Leishmania-infected and bloodfed sand flies fed with sterile $20 \% \mathrm{w} / \mathrm{v}$ sucrose solution via cotton wool. Sand fly Leishmania infections were estimated by dissecting infected sand fly guts at $72 \mathrm{~h}$ after starting Serratia feeding. Bacterial population size was also estimated in the gut by dissecting insects $72 \mathrm{~h}$ post-infection, four pools of 3 midguts were homogenised in $50 \mu$ l of PBS per pool and serial dilutions were inoculated onto LB agar plates and incubated at $26^{\circ} \mathrm{C}$ for $24 \mathrm{~h}$.

\section{Statistical analysis}

Survival analyses were performed using the Kaplan-Meier Log Rank X2 test. Multiple comparisons were carried out with Kruskal-Wallis and pair-wise comparisons using the Mann-Whitney $U$ test and Fishers Exact test. Results are expressed as the group mean \pm SEM. Significance was considered when $p<0.05$. All data were analysed with SPSS Data Editor Software (version 17.0, SPSS Inc).

\section{Results and discussion}

Effect of commensal microorganisms on Leishmania infection of Lutzomyia longipalpis

A wide range of microbial phylotypes have been found associated with adult phlebotomine sand flies collected in the field $[4-7,32]$ and the percentage of field caught females found to contain a Leishmania population in their gut were usually below 1\% [33-35]. Thus Leishmania is only one of many microorganisms vying to occupy the sand fly gut. In the first part of the study we assessed the effect of the interaction between gut microorganisms with Leishmania on subsequent successful colonisation. We used a yeast, Pseudozyma sp. and a bacterium Asaia sp. isolated from the gut of female sand flies collected in a region endemic for visceral leishmaniasis and also $O$. intermedium, present in our sand fly colony (and other colonies [8]). The $\alpha$-proteobacterium Asaia used in these experiments was the first of this genus to be isolated from a New World sand fly; in this case a female Lu. longipalpis from a chicken house in Teresina (Piauí-Brazil). There is one record of Asaia being present in a female Phlebotomus sergentii [36]. Asaia were previously isolated from anopheline mosquitoes and are highly prevalent and abundant in their midgut microbiota [37,38]. It is interesting that both Asaia and the yeast-like fungus Pseudozyma are associated with plants and have osmophilic properties $[39,40]$ and may have been acquired by the female during plant feeding for sugar-rich food.

To investigate the microbe-Leishmania interaction in vivo, female $L u$. longipalpis were given a yeast or a bacterial feed 4 days prior to being membrane-fed with Leishmania mexicana in a bloodmeal. The impact upon the Leishmania population was evaluated by estimating the number of Leishmania promastigotes inside the sand fly gut $72 \mathrm{~h}$ after the bloodmeal and the number of sand flies infected were compared to control flies (Figure 1). Prior colonisation by Asaia and O. intermedium significantly reduced the size of the Leishmania populations within the sand flies (Figure 1A) and with O. intermedium, the sand fly infection rates were also reduced (Figure 1B). We also investigated the effect of pre-feeding a combination of two strains of microorganisms, Pseudozyma with Asaia, and there was a significant reduction both in the size of the Leishmania populations and in the number of sand flies containing a Leishmania infection after pre-feeding with the mixed culture (Figure 1A and 1B). This result is consistent with predictions of community ecology theory applied to insect gut microbiota [41] that suggest increasing species diversity within the sand fly gut, by feeding two rather than one species, would lead to a community more resistant to invasion by the Leishmania. It is also possible that the other gut bacteria already present, albeit at low levels (Day 3 onwards: Additional file 1: Figure S2), may have contributed to the colonisation resistance seen towards the Leishmania.

The effects were further studied by comparing two $O$. intermedium concentrations of $10^{6}$ and $10^{7} \mathrm{cfu} \mathrm{mL}^{-1} \mathrm{fed}$ to female $\mathrm{Lu}$. longipalpis 4 days prior to being fed with L. mexicana. The results showed that there was no concentration dependent effect as the Leishmania promastigote population was not reduced when flies were membrane-fed with O. intermedium at $10^{7} \mathrm{CFU} \mathrm{mL}^{-1}$, in comparison with flies fed the lower concentration of $10^{6} \mathrm{CFU} \mathrm{mL} \mathrm{m}^{-1}$ (Figure 2A). A sand fly is estimated to ingest less than $0.8 \mu \mathrm{l}$ of fluid in a meal [19], this suggests that an initial dose of $<800$ bacteria was sufficient to reduce the subsequent Leishmania population in sand flies. 
A

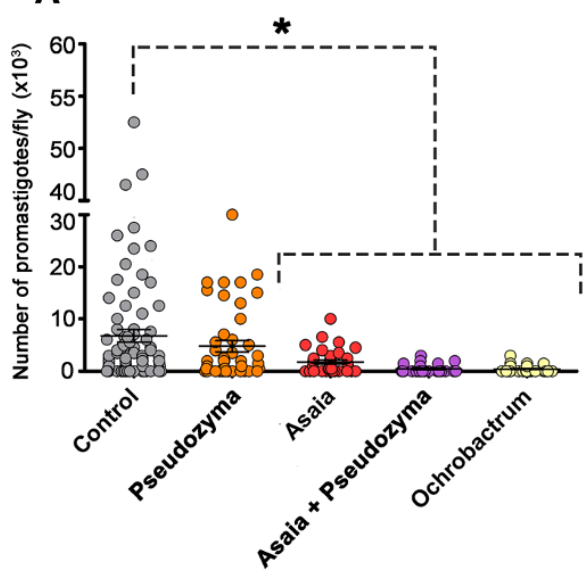

B

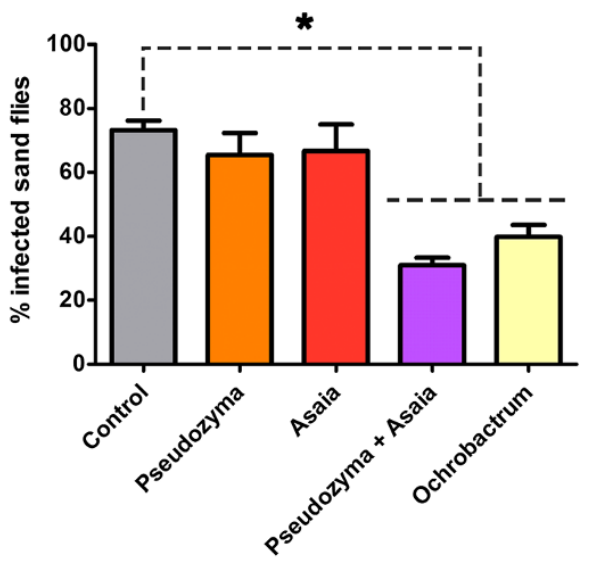

Figure 1 Impact of pre-feeding bacteria and yeast on the subsequent Leishmania population within the gut of female Lu. longipalpis. (A) Leishmania promastigote population estimated within the midgut of Lu. Iongipalpis after feeding with Pseudozyma sp; Asaia sp. or O. intermedium; $2-4 \times 10^{7} \mathrm{CFU} \mathrm{mL} \mathrm{m}^{-1}$. Circles represent individual parasite counts in individual sand fly midguts from 3 independent experiments for each microbial species. *Kruskal-Wallis: $P \leq 0.0001$. Mann-Whitney $U$ test: $P \leq 0.007$. (B) Percentage of female flies infected with Leishmania. Control group were fed on $7 \% \mathrm{w} / \mathrm{v}$ sucrose before being fed with Leishmania. *Fisher's Exact Test $\mathrm{P} \leq 0.0001$.

We also showed that the Ochrobactrum needed to be alive to exert these effects, as the Leishmania infection rates observed in sand flies fed with heat-inactivated bacteria at $10^{7} \mathrm{CFU} \mathrm{mL} \mathrm{m}^{-1}$ were not significantly different from the controls (Figure 2B). This result together with the observation that only bacterial or yeast cells, and not supernatant, showed growth limiting effects towards Leishmania in vitro (Additional file 2: Figure S1A \& B) suggest that microbial interference in Leishmania development is likely to occur only when the live cells are present.

The sand flies used in the colonisation study were reared in an aseptic environment with minimal microbial contamination but were not "germ free". The aseptically prepared adults were assessed using QPCR for the bacterial 16S rDNA gene and the results indicated a low level
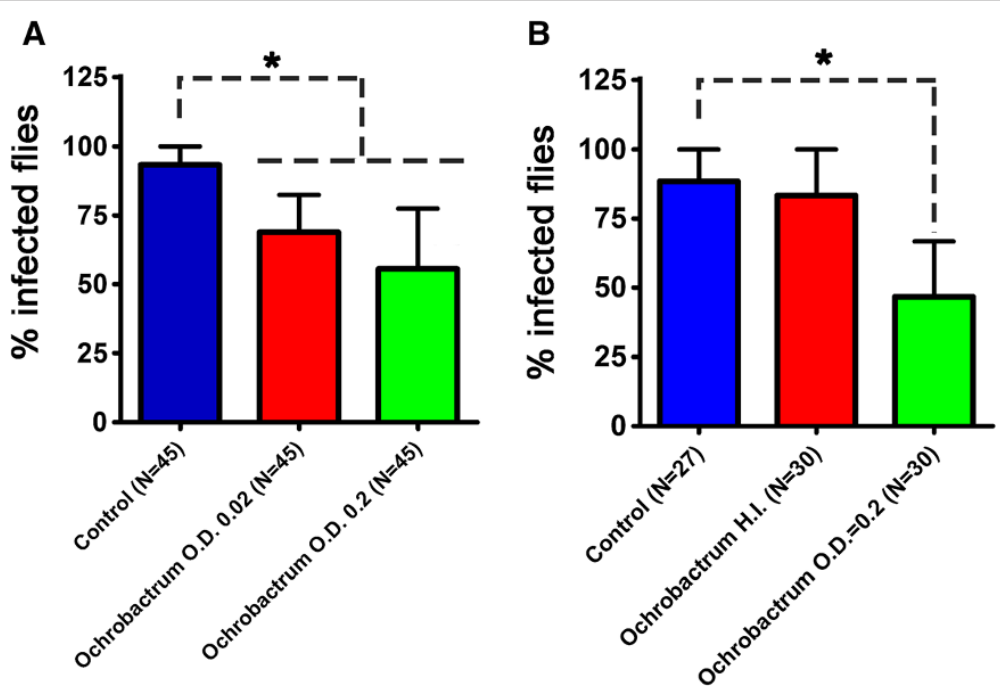

Figure 2 Effect of $O$. intermedium concentration and heat inactivation on the percentage of female Lu. Longipalpis containing a Leishmania infection. (A) Two concentrations of O. intermedium of $10^{6}$ (OD 0.02) and $10^{7} \mathrm{CFU} \mathrm{mL}^{-1}$ (OD 0.2) were used to feed groups of sand flies prior to Leishmania infection. Control group were fed on $7 \%$ sucrose only before infections. Three independent experiments were carried out. Fisher's Exact Test:* $P \leq 0.0001$. (B) Effect of feeding heat inactivated (HI) O. intermedium $\left(10^{7} \mathrm{CFU} \mathrm{mL}^{-1}\right)$ on subsequent Leishmania percentage infection of females. Infection rates from individual sand fly midguts from 3 independent experiments. Fisher's Exact Test: ${ }^{*} P \leq 0.0001$. 
of bacteria associated with these insects that did not change significantly after bloodfeeding (Additional file 1: Figure S2). Newly emerged adults were associated with significantly larger amounts of bacterial DNA that were not evident in 3 day old adults. We suggest that the bacterial DNA was associated with the meconium [42] that would have contained bacteria (dead or alive) as remnants of the larval gut. The meconium is voided from the adult gut within hours of the adult emerging from the pupae. Therefore, the sand flies may have contained gut bacteria carried over from the larval stages after pupation or from environmental contamination. Antibiotic treatment to eliminate the gut bacteria was not used as relevant antibiotics would potentially also inhibit Leishmania development. Germ free insects were not used as the immune response of these insects may differ markedly from a conventionally reared insect [43] and the immune response may form a vital function as part of the interaction between sand fly host, Leishmania and bacteria or yeast $[23,44]$.

The majority of the studies describing the effects of gut microbiota on parasite prevalence and development within medically important insect vectors have been done with mosquitoes [45], tsetse flies [13] and triatomine bugs [12]. Plasmodium is only found within the mosquito midgut for a limited period of time in comparison to Leishmania in the gut of their insect vector. However, a positive correlation was observed between the presence of a midgut microbiota and inhibition of Plasmodium development within the mosquito [43]. This inhibition was variously attributed to direct competition [43], active production of ROS by indigenous mosquito microbiota [46] or a consequence of activation of the basal immunity generated by mosquito gut bacteria [47]. Although very dominant in anopheline microbiota, there is no evidence that the symbiotic Asaia induce colonisation resistance in mosquitoes [38].
Our previous studies indicate that immune activation of the sand fly can lead to loss of Leishmania infection [23] and that although Leishmania do not appear to activate a ROS response, bacteria in the gut do cause ROS activation [44]. Direct production of antimicrobial compounds by bacteria, as found in other insects [48] cannot be discounted. The mechanisms of colonisation resistance in sand flies are therefore likely to be multifaceted; including direct bacterial-mediated lysis, competition for binding sites and nutrients or indirect via immune priming of the sand fly host. An intriguing parallel situation is demonstrated in plant systems with the epiphytic Pseudozyma and plant pathogen Botrytis cinerea [49]. Pseudozyma aphidis secreted extracellular metabolites not only inhibit the pathogen but also primes the plant immune system to invoke a local and systemic immune response towards the pathogen.

\section{Role of Leishmania in protecting Lutzomyia longipalpis from an insect pathogen}

The final part of the investigation addressed the hypothesis that Leishmania are beneficial to the sand fly host. There are many microbial species encountered by the sand fly vector that are potentially pathogenic to the insect. We investigated circumstances in which the Leishmania may prevent the development of a sand fly pathogen. The colonisation experiments were repeated but the feeding regime was reversed; we fed bacteria to sand flies already colonised with Leishmania. We used the insect bacterial pathogen, Serratia marcescens as it is known to be associated with wild $L u$. longipalpis and is also lethal to Leishmania in vitro [7,25]. Preliminary in vitro experiments confirmed that both the cells of $S$. marcescens and the spent culture media incubated with our strain of $L$. mexicana led to suppression of parasite growth (Figure 3).

Flies that were blood fed and then subjected to a daily feed of Serratia in a sugar meal (Figure 4A) succumbed
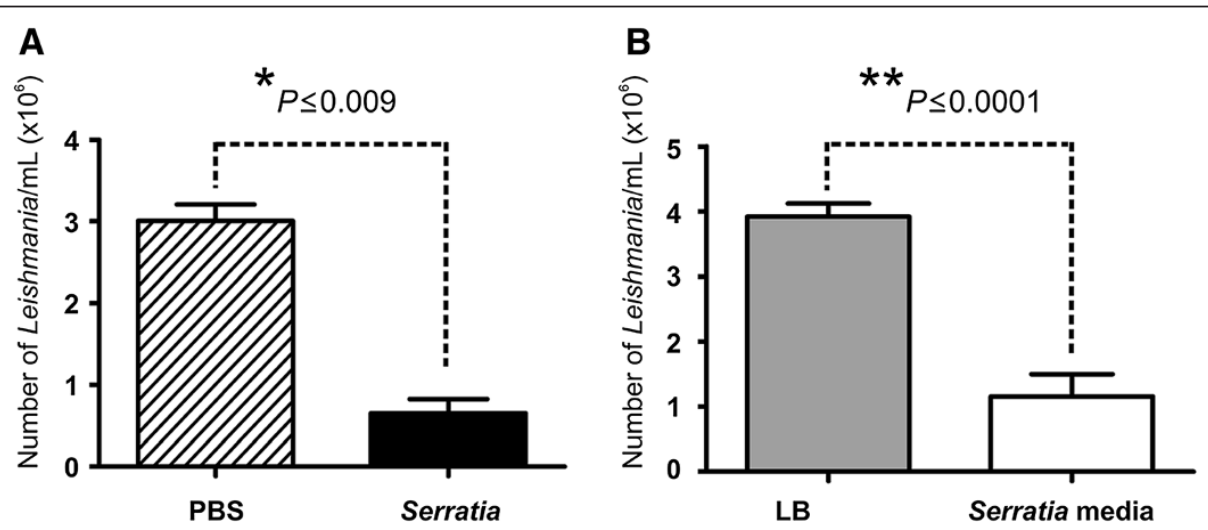

Figure 3 Effect of $\mathbf{S}$. marcescens on growth of $\mathbf{L}$. mexicana in vitro. (A) In vitro 24 h incubation of Serratia bacterial cells (10 ${ }^{7}$ CFU $\mathrm{mL}^{-1}$ ) or (B) filtered spent medium from Serratia culture with L. mexicana $\left(3 \times 10^{6}\right.$ promastigotes $\left.\mathrm{mL}^{-1}\right)$. Results are based on triplicate samples repeated three times and bar charts represent mean \pm SEM. ${ }^{*} P \leq 0.009$. ${ }^{*} P \leq 0.0001$ (Mann-Whitney $U$ test). 


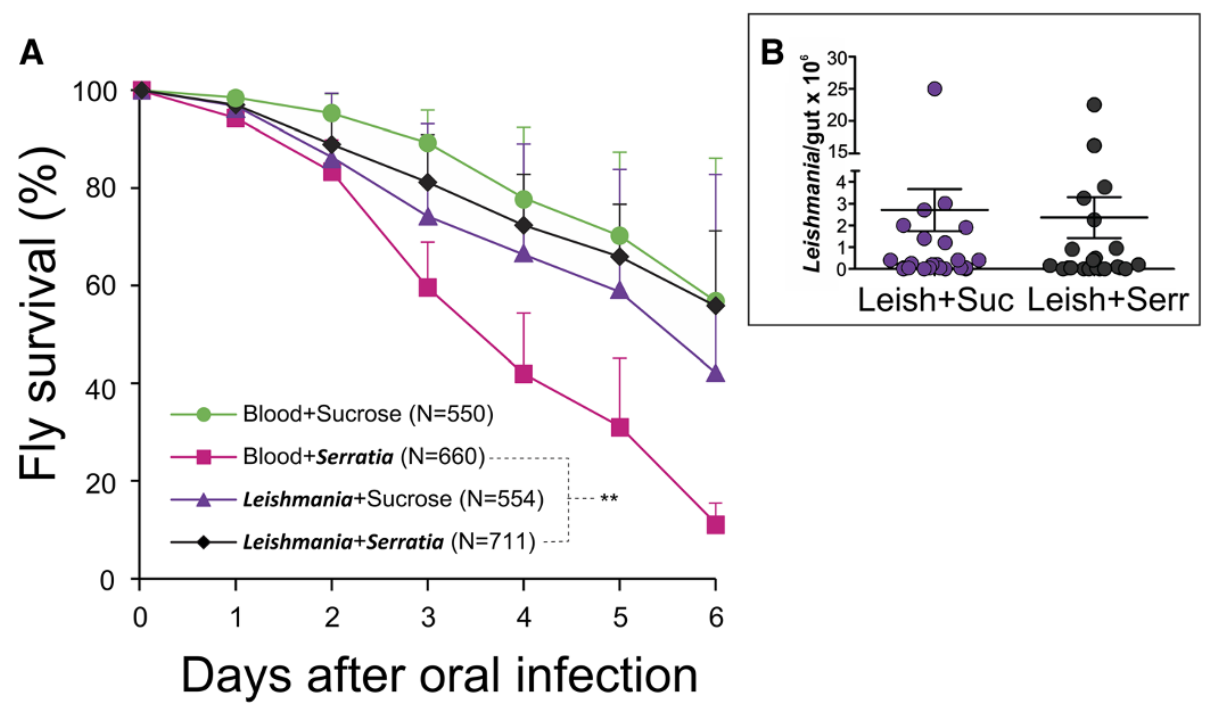

Figure 4 Effect of Leishmania infection on sand fly survival after oral challenge with Serratia marcescens. (A) Survival of female Lu. longipalpis containing Leishmania after oral challenge with Serratia marcescens in sucrose (diamond) in comparison with insects fed with a bloodmeal containing Serratia (square), Leishmania (triangle), or blood followed by sucrose (circle). ${ }^{* *} P \leq 0.0001$; Chi-square 96.987 (Log Rank-Mantel Cox). (B) Scatter plot showing Leishmania promastigote population, at day 3, within individual sand fly midguts after

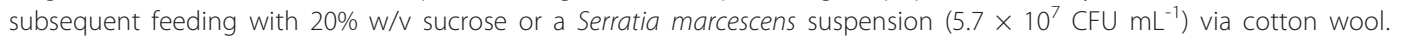

to the bacterial infection and only $11 \%$ survived after 6 days. In contrast, sand fly survival was significantly higher $(56 \%$ after 6 days; Figure 4A) when fed a blood meal containing L. mexicana amastigotes and then subjected to a daily feed of Serratia in a sugar meal. The presence of Leishmania in the gut enhanced the survival of Serratia-challenged sand flies in comparison with those not infected with Leishmania. Remarkably, the population of Leishmania in these sand flies was not significantly different to those in a further set of control flies that were not fed Serratia (Figure 4B, Additional file 3: Figure S3). Survival of sand flies infected with Leishmania but not challenged with Serratia was no different to that of control sand flies (Figure 4A).

When an insect vector containing a medically important parasite is exposed to a pathogen of the vector it threatens the successful transmission of the parasite. In our experiments when the Leishmania infected sand flies were subsequently fed with Serratia, the Leishmania population was similar to that of the control insects. This suggests that the association between Leishmania and its insect vector promote both survival of the insect

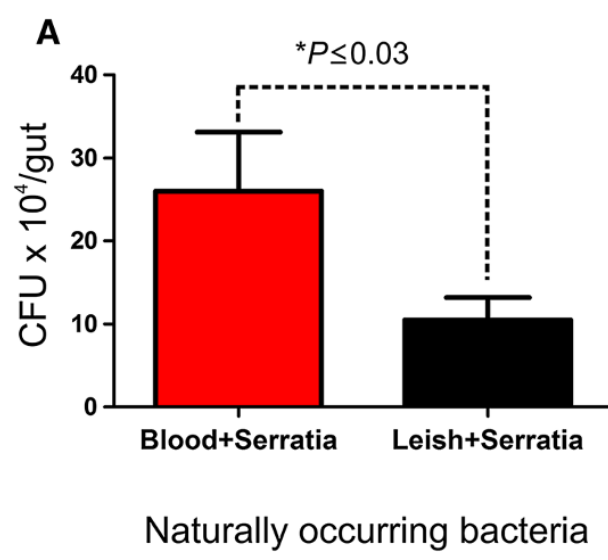

B

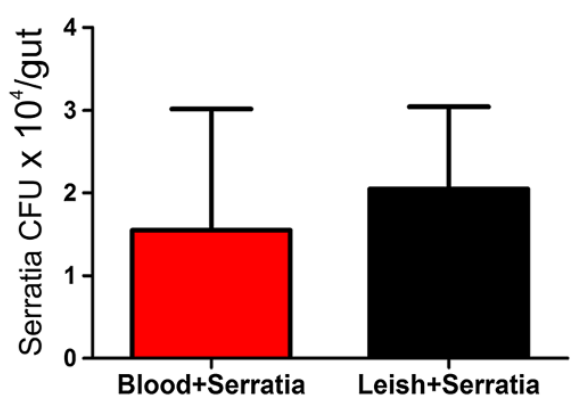

\section{Serratia}

Figure 5 Estimate of naturally-occurring sand fly gut bacteria. (A) and Serratia marcescens (B) in Leishmania infected flies after oral challenge with Serratia. Estimated as CFUs present in individual Lu. longipalpis midguts either uninfected (Blood + Serratia) or infected with L. mexicana (Leish + Serratia) at 3 days after daily oral challenge with Serratia marcescens via cotton wool $\left(5.7 \times 10^{7}\right.$ Serratia CFUmL ${ }^{-1}$, resuspended with sterile $20 \%$ w/v sucrose solution). Asterisk represents statistical difference using Mann-Whitney $U$ test $(P \leq 0.03)$ of at least two independent experiments. 
and the flagellate population. Additionally there was no difference in the survival of flies with Leishmania infection with or without the Serratia challenge during the period of the experiment.

There was a significant reduction in the population of naturally-occurring sand fly gut bacteria sampled at 3 days (Figure 5A) in Leishmania infected sand flies that had been exposed to a daily Serratia oral challenge. However, there were no differences in Serratia populations in surviving flies infected with Leishmania in comparison with the flies without Leishmania (Figure 5B). The decrease in gut microbiota is consistent with the idea that colonisation resistance was generated by Leishmania towards indigenous bacteria within the sand fly. It should be noted that sampling of blood fed sand flies without Leishmania may have resulted in a lower than expected population of Serratia since flies with higher Serratia doses probably died before the time of sampling.

These experiments highlighted one circumstance in which the association between Leishmania and sand flies may be mutually beneficial. Increased protection from insect pathogens will extend the lifespan of sand flies. But does the potential benefit of the Leishmania help to offset the known fitness costs [18] of a Leishmania population in the sand fly gut? This is a significant question since even a few days life extension in a disease endemic area may greatly promote Leishmania transmission and contribute to the successful spread of the human disease. An increased disease resistance conferred on the insect by Leishmania could be an important evolutionary pressure for the maintenance of the Leishmania-vector association. Sand flies more resistant to Leishmania infection may be more exposed to enteric bacterial entomopathogens. In this case maintenance of a small fraction of Leishmaniasusceptible flies in the vector species may ensure insect survival within a population that is succumbing to an entomopathogen.

A further implication of the protective effect of Leishmania is that implementing a biological control campaign against insect vectors using insect pathogens may have unwarranted effects. Sand flies not carrying Leishmania may succumb more rapidly to the biological control agent and this would lead to the development of a wild sand fly population containing an increased proportion of the surviving flies carrying the human disease agent. Any new forms of control aimed at insect vectors of human disease need to consider the total macro- and micro- ecology of the relationship between the insect and the human parasite.

\section{Conclusions}

The colonisation experiments show that pre-feeding yeast or bacteria to the sand fly can prevent the establishment of Leishmania within the sand fly vector. There are many factors governing the prevalence of leishmaniasis within endemic areas [50], but the possibility that the species composition and abundance of microbiota in the female sand fly may influence Leishmania transmission should be considered in modelling the spread of the disease.

Our study also showed that sand flies infected with Leishmania were able to survive an attack by a bacterial pathogen that would have killed the insect and we concluded that Leishmania may benefit its insect host whilst increasing the potential to establish itself in the sand fly vector. We suggest that this is evidence for a mutually beneficial interaction between the vector fly and Leishmania. Furthermore, this may be an example of a subtle and intermittent interaction existing in the natural habitat that provides an evolutionary pressure for the maintenance of the sand fly - Leishmania association.

\section{Additional files}

Additional file 1: Figure S2. Bacterial DNA copy number in newly emerged and aseptically reared sugar-fed (3 day old) and blood fed (BF) female Lu. longipalpis. Bars represent bacterial copy number of 6 pools of 5 ethanol-sterilised and PBS-washed sand flies. Asterisk represents statistical difference (Mann-Whitney $U$ test, $P \leq 0.05$ ).

Additional file 2: Figure S1. Effect of In vitro incubation of yeast and bacterial cells and media on the growth of L. mexicana. (A) Number of L. mexicana promastigotes after in vitro incubation with Pseudozyma sp., Ochrobactrum intermedium and Asaia sp. $\left.\left(10^{7} \mathrm{CFU} \mathrm{mL}\right)^{-1}\right)$ and (B) microbiological media for $24 \mathrm{~h}$ at $26^{\circ} \mathrm{C}$. PBS and LB media were used as a control. Experiments were done in triplicate and repeated three times. Mann Whitney $U$ test: $a-P<0.044$; $b-P<0.001$; $c-P<0.0045$

Additional file 3: Figure S3. Leishmania minicircle kDNA copy number determined by qPCR. Presence in 12 pools of 5 sand flies infected with L. mexicana and subsequently fed with $20 \% \mathrm{w} / \mathrm{v}$ sucrose (Leish + sucrose) or a Serratia suspension (Leish + Serratia $-5.7 \times 10^{7} \mathrm{CFUmL}^{-1}$ prepared in autoclaved $20 \% \mathrm{~W} / \mathrm{V}$ sucrose ) via cotton wool for 6 days. Bar charts represent mean \pm SEM of three independent experiments $(P>0.05)$.

\section{Competing interests}

The authors declare that they have no competing interests.

\section{Authors' contributions}

Conceived and designed the experiments: MRVS, FAG, VMD, PAB, RJD.

Performed the experiments: MRVS, HDA, WSS, FAG, KAM, RRC, RJD. Analyzed the data: MRVS, HDA, RJD. Contributed reagents/materials/analysis tools: RJD, KAM, RRC, PAB. Wrote the paper: MRVS, FAG, PAB, VMD, RJD. All authors read and approved the final manuscript.

\section{Acknowledgements}

The authors would like to thank Ms Davina Moor and Ms Sue Pritchard for technical assistance, Peter Matthews and Jameel Bharmal for assistance with bacterial identification.

This work was funded by UK and Brazilian agencies; the Leverhulme Trust (ref F/00 808/C), Sir John Fisher Foundation, CONACYT, FAPERJ, CNPq (INCT-EM) and CAPES (BEX 5287/09-0) in Brazil.

\section{Author details}

${ }^{1}$ Faculty of Health and Medicine, Division of Biomedical and Life Sciences, Lancaster University, Lancaster, UK. ${ }^{2}$ Present Address: Departamento de Parasitologia, ICB/UFMG, Belo Horizonte, Brazil. ${ }^{3}$ Instituto Oswaldo Cruz, Fundação Oswaldo Cruz, Rio de Janeiro, Brazil. ${ }^{4}$ Departamento de Parasitologia, ICB/UFMG, Belo Horizonte, Brazil. ${ }^{5}$ Liverpool School of Tropical Medicine, Vector Group, Liverpool, UK. ${ }^{6}$ Parasitologia e Microbiologia, CCS, Universidade Federal do Piauí, Teresina, Piauí, Brazil. 'Institute of Integrative Biology, University of Liverpool, Liverpool, UK. 
Received: 1 May 2014 Accepted: 3 July 2014

Published: 23 July 2014

\section{References}

1. Killick-Kendrick R: The biology and control of phlebotomine sand flies. Clin Dermatol 1999, 17(3):279-289.

2. Ready PD: Biology of phlebotomine sand flies as vectors of disease agents. Annu Rev Entomol 2013, 58(1):227-250.

3. Bates PA: Leishmania sand fly interaction: progress and challenges Curr Opin Microbiol 2008, 11(4):340-344.

4. Dillon RJ, el Kordy E, Shehata M, Lane RP: The prevalence of a microbiota in the digestive tract of Phlebotomus papatasi. Ann Trop Med Parasitol 1996, 90(6):669-673.

5. Sant'anna MR, Darby AC, Brazil RP, Montoya-Lerma J, Dillon VM, Bates PA, Dillon RJ: Investigation of the bacterial communities associated with females of Lutzomyia sand fly species from South America. PLoS One 2012, 7(8):e42531.

6. Hillesland H, Read A, Subhadra B, Hurwitz I, McKelvey R, Ghosh K, Das P, Durvasula R: Identification of aerobic gut bacteria from the kala azar vector, Phlebotomus argentipes: a platform for potential paratransgenic manipulation of sand flies. Am J Trop Med Hyg 2008, 79(6):881-886.

7. McCarthy CB, Diambra LA, Rivera Pomar RV: Metagenomic analysis of taxa associated with Lutzomyia longipalpis, vector of visceral leishmaniasis, using an unbiased high-throughput approach. PLoS Negl Trop Dis 2011, 5(9):e1304.

8. Volf $\mathrm{P}$, Kiewegova A, Nemec A: Bacterial colonisation in the gut of Phlebotomus duboscqi (Diptera : Psychodidae): transtadial passage and the role of female diet. Folia Parasitol 2002, 49(1):73-77.

9. Lawley TD, Walker AW: Intestinal colonization resistance. Immunology 2013, 138(1):1-11.

10. Dillon RJ, Dillon VM: The gut bacteria of insects: nonpathogenic interactions. Annu Rev Entomol 2004, 49:71-92.

11. Azambuja P, Garcia ES, Ratcliffe NA: Gut microbiota and parasite transmission by insect vectors. Trends Parasitol 2005, 21(12):568.

12. Castro DP, Moraes CS, Gonzalez MS, Ratcliffe NA, Azambuja P, Garcia ES: Trypanosoma cruzi immune response modulation decreases microbiota in Rhodnius prolixus gut and Is crucial for parasite survival and development. PLoS One 2012, 7(5):e36591.

13. Wang J, Weiss BL, Aksoy S: Tsetse fly microbiota: form and function. In Frontiers in cellular and infection microbiology. ; 2013:3.

14. Cirimotich CM, Ramirez JL, Dimopoulos G: Native microbiota shape insect vector competence for human pathogens. Cell Host Microbe 2011, 10(4):307-310

15. Schlein Y, Polacheck I, Yuval B: Mycoses, bacterial infections and antibacterial activity in sandifies (Psychodidae) and their possible role in the transmission of leishmaniasis. Parasitology 1985, 90(01):57-66.

16. Casanova C, Natal D, Santos FAM: Survival, population size, and gonotrophic cycle duration of Nyssomyia neivai (Diptera: Psychodidae) at an endemic area of American cutaneous leishmaniasis in southeastern Brazil. J Med Entomol 2009, 46(1):42-50.

17. Rogers ME, Ilg T, Nikolaev AV, Ferguson MAJ, Bates PA: Transmission of cutaneous leishmaniasis by sand flies is enhanced by regurgitation of fPPG. Nature 2004, 430(6998):463-467

18. Rogers ME, Bates PA: Leishmania manipulation of sand fly feeding behavior results in enhanced transmission. Plos Pathogens 2007, 3:818-825.

19. Dillon R, Lane RP: Influence of Leishmania infection on blood-meal digestion in the sandflies Phlebotomus papatasi and Phlebotomus langeroni. Parasitol Res 1993, 79(6):492-496.

20. Sant'Anna M, Diaz-Albiter H, Mubaraki M, Dillon R, Bates P: Inhibition of trypsin expression in Lutzomyia longipalpis using RNAi enhances the survival of Leishmania. Parasit Vectors 2009, 2(1):62.

21. Telleria EL, Pitaluga AN, Ortigao-Farias JR, de Araujo AP, Ramalho-Ortigao JM, Traub-Cseko YM: Constitutive and blood meal-induced trypsin genes in Lutzomyia longipalpis. Arch Insect Biochem Physiol 2007, 66(2):53-63.

22. Rogers ME, Hajmova M, Joshi MB, Sadlova J, Dwyer DM, Volf P, Bates PA: Leishmania chitinase facilitates colonization of sand fly vectors and enhances transmission to mice. Cell Microbiol 2008, 10(6):1363-1372.

23. Telleria EL, Sant'Anna MR, Ortigao-Farias JR, Pitaluga AN, Dillon VM, Bates PA, Traub-Cseko YM, Dillon RJ: Caspar-like gene depletion reduces
Leishmania infection in sand fly host Lutzomyia longipalpis. J Biol Chem 2012, 287(16):12985-12993.

24. Harhay MO, Olliaro PL, Costa DL, Costa $\mathrm{CH}$ : Urban parasitology: visceral leishmaniasis in Brazil. Trends Parasitol 2011, 27(9):403-409.

25. Moraes CS, Seabra SH, Castro DP, Brazil RP, de Souza W, Garcia ES, Azambuja P: Leishmania (Leishmania) chagasi interactions with Serratia marcescens: ultrastructural studies, lysis and carbohydrate effects. Exp Parasitol 2008, 118(4):561-568.

26. Modi GB, Tesh RB: A simple technique for mass rearing Lutzomyia longipalpis and Phlebotomus papatasi (Diptera: Psychodidae) in the laboratory. J Med Entomol 1983, 20(5):568-569.

27. Webster G, Parkes RJ, Cragg BA, Newberry CJ, Weightman AJ, Fry JC: Prokaryotic community composition and biogeochemical processes in deep subseafloor sediments from the Peru Margin. FEMS Microbiol Ecol 2006, 58(1):65-85.

28. Kurtzman C, Robnett C: Identification and phylogeny of ascomycetous yeasts from analysis of nuclear large subunit (26S) ribosomal DNA partial sequences. Antonie Van Leeuwenhoek 1998, 73(4):331-371.

29. Sant'Anna MRV, Alexander B, Bates PA, Dillon RJ: Gene silencing in phlebotomine sand flies: Xanthine dehydrogenase knock down by dsRNA micro-injections. Insect Biochem Mol Biol 2008, 38(6):652-660.

30. Nicolas LPE, Lang T, Milon G: Real-time PCR for detection and quantitation of Leishmania in mouse tissues. J Clin Microbio/ 2002, 40(4):1666-1669.

31. Nadkarni MA, Martin FE, Jacques NA, Hunter N: Determination of bacterial load by real-time PCR using a broad-range (universal) probe and primers set. Microbiol 2002, 148(1):257-266.

32. Gouveia C, Asensi MD, Zahner V, Rangel EF, Oliveira SM: Study on the bacterial midgut microbiota associated to different Brazilian populations of Lutzomyia longipalpis (Lutz \& Neiva) (Diptera: Psychodidae). Neotrop Entomol 2008, 37(5):597-601.

33. Murray HW, Berman JD, Davies CR, Saravia NG: Advances in leishmaniasis. Lancet 2005, 366(9496):1561-1577.

34. Rangel EF, Lainson R: Proven and putative vectors of American cutaneous leishmaniasis in Brazil: aspects of their biology and vectorial competence. Mem Inst Oswaldo Cruz 2009, 104(7):937-954.

35. Felipe IM AD, Kuppinger O, Santos MD, Rangel ME، Barbosa DS, Barral A Werneck GL, Caldas Ade J: Leishmania infection in humans, dogs and sandflies in a visceral leishmaniasis endemic area in Maranhão, Brazil. Mem Inst Oswaldo Cruz 2011, 106(2):207-211.

36. Akhoundi M, Bakhtiari R, Guillard T, Baghaei A, Tolouei R, Sereno D, Toubas D, Depaquit J, Abyaneh MR: Diversity of the bacterial and fungal microflora from the midgut and cuticle of Phlebotomine sand flies collected in North-Western Iran. PLoS One 2012, 7(11):e50259.

37. Favia G, Ricci I, Damiani C, Raddadi N, Crotti E, Marzorati M, Rizzi A, Urso R, Brusetti L, Borin S, Mora D, Scuppa P, Pasqualini L, Clementi E, Genchi M, Corona S, Negri I, Grandi G, Alma A, Kramer L, Esposito F, Bandi C, Sacchi L, Daffonchio D: Bacteria of the genus Asaia stably associate with Anopheles stephensi, an Asian malarial mosquito vector. Proc Natl Acad Sci U S A 2007, 104(21):9047-9051

38. Damiani C, Ricci I, Crotti E, Rossi P, Rizzi A, Scuppa P, Capone A, Ulissi U, Epis S, Genchi M, Sagnon N, Faye I, Kang A, Chouaia B, Whitehorn C, Moussa GW, Mandrioli M, Esposito F, Sacchi L, Bandi C, Daffonchio D, Favia G: Mosquito-bacteria symbiosis: the case of Anopheles gambiae and Asaia. Microb Ecol 2010, 60(3):644-654

39. Crotti E, Damiani C, Pajoro M, Gonella E, Rizzi A, Ricci I, Negri I, Scuppa P, Rossi P, Ballarini P, Raddadi N, Marzorati M, Sacchi L, Clementi E, Genchi M, Mandrioli M, Bandi C, Favia G, Alma A, Daffonchio D: Asaia, a versatile acetic acid bacterial symbiont, capable of cross-colonizing insects of phylogenetically distant genera and orders. Environ Microbiol 2009, 11(12):3252-3264.

40. Wei YH, Lee FL, Hsu WH, Chen SR, Chen CC, Wen CY, Lin SJ, Chu WS, Yuan GF, Liou GY: Pseudozyma antarctica in Taiwan: a description based on morphological, physiological and molecular characteristics. Bot Bull Acad Sin 2005, 46(3):223-229.

41. Dillon RJ, Vennard CT, Buckling A, Charnley AK: Diversity of locust gut bacteria protects against pathogen invasion. Ecol Lett 2005, 8(12):1291-1298.

42. Moll RM, Romoser WS, Modrzakowski MC, Moncayo AC, Lerdthusnee K: Meconial peritrophic membranes and the fate of midgut bacteria during mosquito (Diptera: Culicidae) metamorphosis. J Med Entomol 2001, 38(1):29-32. 
43. Dong Y, Manfredini F, Dimopoulos G: Implication of the mosquito midgut microbiota in the defense against malaria parasites. PLoS Pathog 2009, 5(5):e1000423.

44. Diaz-Albiter H, Sant'Anna MR, Genta FA, Dillon RJ: Reactive oxygen species-mediated immunity against Leishmania mexicana and Serratia marcescens in the sand phlebotomine fly Lutzomyia longipalpis. J Biol Chem 2012, 287(28):23995-24003.

45. Gendrin M, Christophides GK: The Anopheles Mosquito Microbiota and Their Impact on Pathogen Transmission. In Anopheles mosquitoes - New insights into malaria vectors. Edited by Manguin S. Croatia: InTech Europe; 2013. DOl: $10.5772 / 55107$.

46. Cirimotich CM, Dong YM, Clayton AM, Sandiford SL, Souza-Neto JA, Mulenga M, Dimopoulos G: Natural Microbe-Mediated Refractoriness to Plasmodium Infection in Anopheles gambiae. Science 2011, 332(6031):855-858.

47. Cirimotich CM, Dong Y, Garver LS, Sim S, Dimopoulos G: Mosquito immune defenses against Plasmodium infection. Dev Comp Immunol 2010, 34(4):387-395.

48. Dillon RJ, Charnley AK: Mutualism between the desert locust Schistocerca gregaria and its gut microbiota. Res Microbiol 2002, 153(8):503-509.

49. Buxdorf K, Rahat I, Gafni A, Levy M: The epiphytic fungus Pseudozyma aphidis induces jasmonic acid-and salicylic acid/nonexpressor of PR1-independent local and systemic resistance. Plant Physiol 2013 161(4):2014-2022.

50. Soares MR, Carvalho CC, Silva LA, Lima MS, Barral AM, Rebelo JM, Pereira SR: Molecular analysis of natural infection of Lutzomyia longipalpis in an endemic area for visceral leishmaniasis in Brazil. Cad Saude Publ 2010, 26(12):2409-2413.

doi:10.1186/1756-3305-7-329

Cite this article as: Sant'Anna et al: Colonisation resistance in the sand fly gut: Leishmania protects Lutzomyia longipalpis from bacterial infection. Parasites \& Vectors 2014 7:329.

\section{Submit your next manuscript to BioMed Central and take full advantage of:}

- Convenient online submission

- Thorough peer review

- No space constraints or color figure charges

- Immediate publication on acceptance

- Inclusion in PubMed, CAS, Scopus and Google Scholar

- Research which is freely available for redistribution 